\title{
Esforço de escavação e teor de lipídios em rainhas da formiga cortadeira Atta sexdens rubropilosa
}

\author{
Digging effort and lipid content in leaf-cutting ant queens Atta sexdens rubropilosa
}

\author{
Roberto da Silva Camargo ${ }^{*}$ Luiz Carlos Forti $^{\mathrm{I}}$
}

\section{RESUMO}

As formigas cortadeiras, importantes pragas agrícolas, anualmente realizam a fundação de novas colônias. Para o início de novas colônias, é preciso a escavação de túneis $e$ de câmaras no solo, sendo que, para isso, as rainhas recém fecundadas gastam muita energia, no entanto a quantificação dessa energia gasta é desconhecida. Nossa hipótese é que a reserva lipídica, armazenada no corpo da rainha, seja utilizada durante a escavação do ninho. Testamos essa hipótese comparando o aumento do esforço de escavação das rainhas, as quais foram experimentalmente estimuladas a escavar uma, duas ou três vezes consecutivas, em relação ao controle, ou seja, em relação às rainhas que não escavaram. Foram quantificados a massa corporal e o teor de lipídios das rainhas. Os resultados mostraram que, ao contrário de nossas expectativas iniciais, massa e teor de lípideos não foram afetadas pelo aumento do esforço de escavação do ninho. Conclui-se que a escavação pelas rainhas não afetou os percentuais de lipídios em seu corpo, consequentemente, o conteúdo de energia. Provavelmente, os recursos energéticos para a escavação não se originam de fontes lipídicas, mas de outros, provavelmente carboidratos.

Palavras-chave: teor de lipídeos, gasto energético, saúvas, fundação de colônias.

\section{ABSTRACT}

Nest digging spends a lot of energy existing a question about how much energy is required to dig the tunnel and initial chamber. It was hypothesized that the lipid content is used during nest digging. This hypothesis was tested by comparing the consequences of increasing digging effort in queens that were experimentally stimulated to excavate a complete founding nest either once, twice or three times consecutively, compared to control queens that didn't dig. Weight and lipid content of queens were quantified. Results showed that, in contrast with the initial expectations, weight and lipid content were not affected by the increased digging effort in the experimentally-induced successive excavations. It was conclude that the excavation by the queens did not affect the percentages of lipids and consequently the energy content, in their bodies. Probably, energy resources for the excavation wasn't originated from lipid reserves, but from other energy sources, perhaps carbohydrates.

Key words: lipid content, energy-consuming, ant, Atta, nest founding.

\section{INTRODUÇÃO}

As formigas cortadeiras, importantes pragas agrícolas, anualmente realizam a fundação de novas colônias. Para o início de novas colônias é preciso a escavação de túneis e de câmaras no solo. As rainhas de Atta sexdens (Linnaeus) têm aproximadamente $40 \%$ de sua massa corporal composta em lipídeos (SEAL, 2009) e possuem reservas energéticas suficientes para manter a sua taxa de produção de prole alta por várias semanas (CAMARGO et al., 2011). Nesse sentido, a rainha perde cerca de $40 \%$ da sua massa corporal após 60 dias (DELLA LUCIA et al., 1995), atingindo sua massa corporal mínima, antes que as primeiras operárias comecem a forragear. Quando o forrageamento é iniciado, a rainha recupera a sua massa corporal, devido à alimentação dentro da colônia (DELLA LUCIA et al., 1990). Entretanto, antes da fase claustral, a rainha tem que escavar seu próprio ninho. Essa atividade de escavação do túnel e da câmara inicial leva em média cerca de 6 a 10 horas (AUTUORI, 1942). Durante a escavação, as rainhas realizam cerca de 300 viagens para empilhar solo escavado, sendo que cada viagem dura de 30

'Laboratório de Insetos Sociais-Praga, Departamento de Produção Vegetal, Faculdade de Ciências Agronômicas, Universidade Estadual Paulista (UNESP), 18603-970, CP 237, Botucatu, SP, Brasil. E-mail: camargobotucatu@yahoo.com.br. *Autor para correspondência. 
segundos a 30 minutos, com velocidade média de $3 \mathrm{~cm}$ hora $^{-1}$ (RIBEIRO, 1972). Devido a essa atividade ser muito intensa, presume-se que o custo energético da escavação seja alto. Até o momento, não existem dados disponíveis na literatura que relatem sobre a quantidade de energia da rainhas que é necessária para a escavação dos ninhos. Dessa forma, postula-se que a energia necessária seja retirada de reservas lipídicas, pois os carboidratos são mais utilizados durante o voo nupcial (JUTSUM \& QUINLAN, 1978; PASSERA et al., 1989). Entretanto, sabe-se que o esforço de escavação afeta significativamente a sobrevivência de rainhas de Atta sexdens rubropilosa (Forel) (CAMARGO et al., 2011). Esses mesmos autores verificaram ainda que o aumento da mortalidade em rainhas que sucessivamente escavaram dois ou três ninhos ocorreu na primeira semana após o esforço. Como consequência, provavelmente, pode-se dizer que a mortalidade da rainha foi causada pelo esgotamento das reservas corporais.

Assim, a hipótese do presente trabalho é que a reserva de lipídeos da rainha recém fecundada seja utilizada durante a escavação do ninho. Testamos essa hipótese pela comparação do esforço de escavação das rainhas que foram experimentalmente estimuladas a escavar um ninho, uma, duas ou três vezes consecutivas, em relação às rainhas que não escavaram.

\section{MATERIAL E MÉTODOS}

Esforço de escavação das rainhas: As rainhas foram coletadas imediatamente após o voo nupcial, na Fazenda Experimental Lageado, FCA / UNESP, Botucatu - Brasil, e, em seguida, foram mantidas individualmente em pequenos recipientes de plástico sem solo ( $11 \mathrm{~cm}$ de diâmetro e $8 \mathrm{~cm}$ de altura), com $1 \mathrm{~cm}$ de gesso úmido no fundo, para posterior determinação do teor de lipídeo $(\mathrm{N}=20)$. Todos os experimentos foram realizados no Laboratório de Insetos Pragas Sociais, FCA / UNESP, Botucatu. Foram acondicionadas à temperatura de $24 \pm 2^{\circ} \mathrm{C}$, umidade relativa de $80 \%$ e um fotoperiodo de 12 horas claro. Cada ensaio foi iniciado colocando-se uma única rainha, imediatamente após o voo núpcial, sobre a superfície de um tubo preenchido com solo ( $25 \mathrm{~cm}$ de altura e $10 \mathrm{~cm}$ de diâmetro), onde a rainha poderia iniciar a sua escavação. $O$ solo utilizado foi coletado no Campus da Faculdade de Ciências Agronômicas (Latossolo), à uma profundidade de 60 $\mathrm{cm}$ (densidade do solo $=1,6 \mathrm{~g} \mathrm{~cm}^{-3}$, teor de água: $5,4 \%$ ). Quatro séries experimentais foram realizadas: A) Sem escavação: as rainhas não escavaram, e permaneceram em recipientes plásticos para posterior determinação de lipídeos ( $\mathrm{N}=20)$. B) Escavação única: as rainhas escavaram no tubo preenchido com o solo, tal como descrito acima, até sua clausura, sendo uma indicação de que elas escavaram a câmara (EIDMANN, 1935) ( $=20)$. C) Escavação dupla: rainhas provinientes da escavação única, depois de finalizar a escavação do ninho, foram imediatamente retiradas da câmara escavada e novamente submetidas a um novo tubo de escavação, paraque começassem a escavar um novo ninho $(\mathrm{N}=20)$. D) Escavação tripla: como descrito acima, mas as rainhas escavaram sucessivamente três vezes $(\mathrm{N}=20)$. Depois de escavar (única ou repetida vezes), as rainhas foram imediatamente colocadas em pequenos recipientes de plástico para determinação do teor de lipídeos. Determinação de lipídeos: Foi utilizada uma adaptação ao método de extração de $\mathrm{COOK}$ et al. (2010), em que os insetos foram imersos em solvente orgânico até atingirem um peso constante. Seguindo essa metodologia, as rainhas foram imersas em $10 \mathrm{ml}$ de pentano durante 24 horas, individualmente fechadas. Após esse período, o solvente foi removido e as rainhas permaneceram durante 24 horas a $70^{\circ} \mathrm{C}$ em uma estufa. Posteriormente, foi determinada sua massa em balança analítica. O procedimento foi repetido por 172 horas de extração. Análise estatística: Os dados da massa e o conteúdo lipídico das rainhas foram comparados pela Análise de Variância e Kruskal-Wallis $(\alpha=0,05)$, utilizando o programa SigmaPlot 11.0.

\section{RESULTADOS}

As massas corporais das rainhas foram em média: 661,09 $\pm 62,15 \mathrm{mg}$ sem escavação,

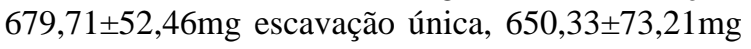

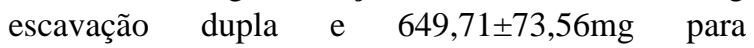
escavação tripla (Figura 1 A). Não foram encontradas diferenças significativas entre as séries experimentais (ANOVA, $\mathrm{F}_{3,79}=0,903, \quad \mathrm{P}=0,444$ ). Em relação ao teor de lípideos das rainhas: $37,15 \%$ sem escavação, 36,66\% escavação simples, 36,63\% escavação dupla e $37,94 \%$ para escavação tripla (Figura 1 B). Não foram encontradas diferenças significativas entre as séries experimentais (KruskalWallis $\mathrm{H}$ teste, $\mathrm{H}=3,183, \mathrm{df}=3, \mathrm{P}=0,364)$.

\section{DISCUSSÃO}

Sob as condições experimentais, rainhas de formigas cortadeiras escavaram o ninho como já foi 


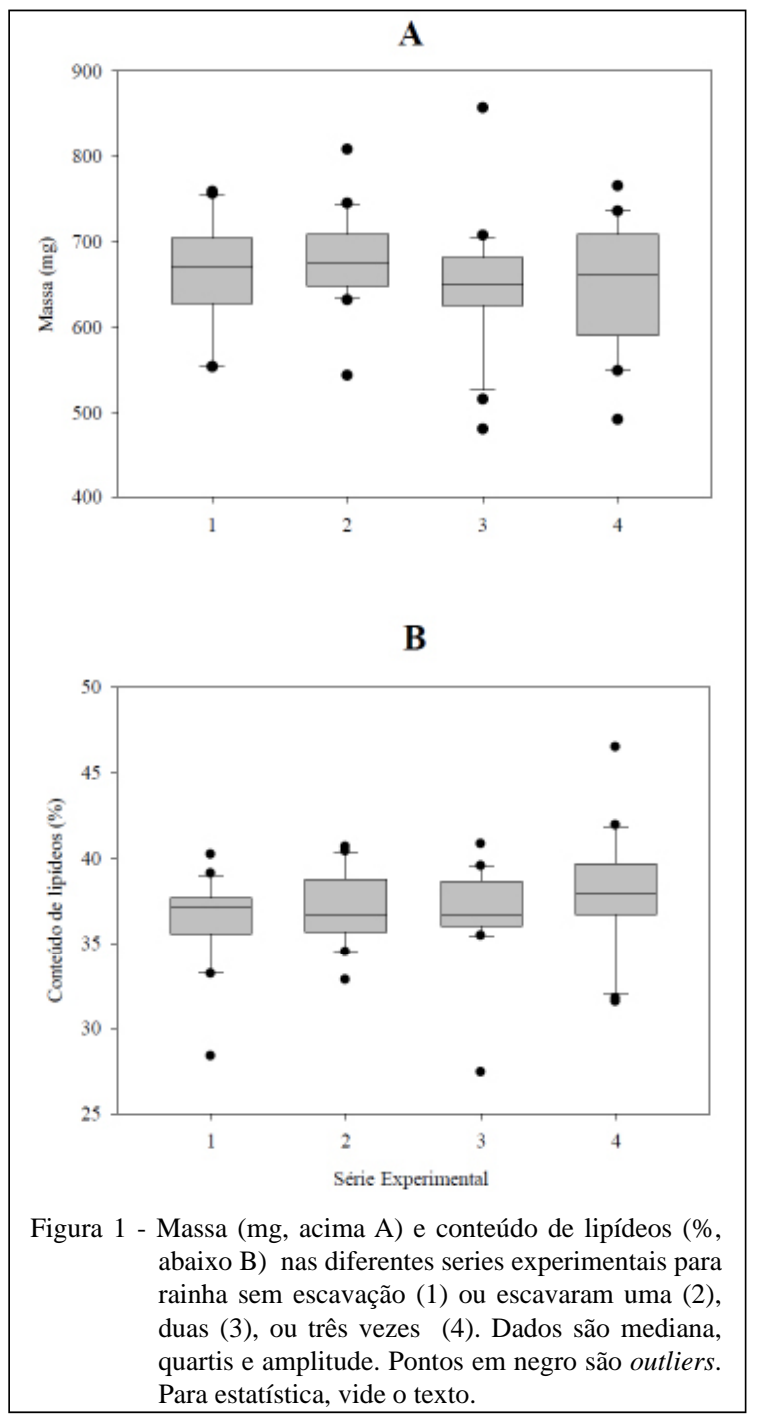

descrito anteriormente na literatura, primeiramente um túnel e, posteriormente, uma câmara (EIDMANN, 1935; CUNHA, 1968; CAMARGO et al., 2011; FUJIHARA et al., 2012). A profundidade da câmara inicial, em torno de 8,5 a $15 \mathrm{~cm}$ (AUTUORI, 1942; RIBEIRO, 1972), é um produto da seleção natural que minimizou o esforço da escavação (CAMARGO et al., 2011) com um ambiente microclimaticamente estável, para um bom desenvolvimento da prole e do jardim de fungo (BOLLAZZI et al., 2008). Esse controle da profundidade ideal ocorre pela rainha por meio autopercepção da distância percorrida e pelo controle temporal durante a escavação do túnel, evitando um gasto excessivo de tempo e investimento energético quando estão escavando o ninho sob diferentes condições ambientais, como estudado para Atta vollenweideri (Forel) (FRÖHLE \& ROCES, 2012). Sob o mesmo ponto de vista de conteúdo energético, essa adaptação da rainha promove economia da energia necessária para todas as atividades vindouras durante a fase claustral.

Além disso, as rainhas apresentam um custo adicional devido à redução da imunidade, em decorrência do acasalamento múltiplo (poliandria). BAER et al. (2006) verificaram na espécie Atta colombica (Guirin-Mineville) que ocorre uma baixa resposta imunológica quando a fêmea apresenta mais espermatozoides em sua espermateca, ocasionando uma maior mortalidade desse grupo de rainhas. Uma maior mortalidade de rainhas de Atta sexdens rubropilosa também ocorre quando essas são expostas a um esforço de escavação intenso, ou seja, escavação de câmaras mais profundas (CAMARGO et al., 2011).

Entretanto, nossos resultados mostram que a massa e o teor de lipídios das rainhas de Atta sexdens rubropilosa não foram afetados pelo aumento do esforço de escavação, ou seja, pelas escavações sucessivas experimentalmente induzidas (Figura 1). Provavelmente, recursos energéticos para a escavação não se originam de reserva lipídica, mas a partir de outra fonte de energia, provavelmente, carboidratos. Por exemplo, em condições laboratóriais, os machos de Atta sexdens tiveram cerca de $21 \%$ da massa corporal consumidas após o voo nupcial, sendo basicamente carboidratos (JUTSUM \& QUINLAN, 1978). Esses mesmos autores descobriram também que os lípideos não são utilizados como recurso energético durante o voo. Da mesma forma, os carboidratos (armazenados como glicogênio) representam a principal fonte de energia para o voo nupcial em Formica lugubris (Zetterstedt) (PASSERA et al., 1989).

Em Cataglyphis cursor (Fonscolombe) e Iridomyrmex humilis (Mayr), os machos apresentam um maior conteúdo de carboidrato, quando comparados com as fêmeas (PASSERA \& KELLER, 1990). A partir desses estudos, podese assumir que o primeiro recurso energético a ser esgotado no voo nupcial é a reserva de carboidratos. Lipídeos e proteínas são de dregadação mais lenta quando comparado com os carboidratos, poupando essas reservas para atividades que seguem após o voo nupcial. Dentre essas atividades, têm-se a oviposição, que não é afetada pelo esforço de escavação repetitivo (CAMARGO et al., 2011), provavelmente, pelo fato de reservas lipídicas serem poupadas e, em insetos, estarem intimamente relacionadas ao processo de ovogênese (CHAPMAN, 1998). Com base em nossos resultados, podemos concluir que a escavação sucessiva pela rainha não afetou os percentuais de lipídeos e, consequentemente, o conteúdo de 
energia, em seu corpo. Em conclusão, nossos estudos com Atta sexdens rubropilosa determinaram que o recurso energético para a escavação não se origina de reserva lipídica, mas a partir de outra fonte de energia, provavelmente, carboidratos.

\section{CONCLUSÃO}

Conclui-se que a escavação sucessiva pela rainha não afetou os percentuais de lipídeos e, consequentemente, o conteúdo de energia, em seu corpo.

\section{AGRADECIMENTOS}

À Fundação de Amparo à Pesquisa do Estado de São Paulo (FAPESP), pelo apoio financeiro aos autores (Processos: 2007/04010-0 e 2007/07091-0). Ao Conselho Nacional de Desenvolvimento Científico e Tecnológico (CNPq), pela bolsa produtividade, em razão da pesquisa 301917/2009-4.

\section{REFERENCES}

AUTUORI, M. Contribuição para o conhecimento da saúva (Atta spp - Hymenoptera- Formicidae). Arquivos do Instituto Biológico, v.13, p.137-150, 1942.

BOLLAZZI, M. et al. Soil temperature, digging behaviour, and the adaptive value of nest depth in South America species of Acromyrmex leaf-cutting ants. Oecologia, v.158, p.165175, 2008. Disponível em: <http://www.ncbi.nlm.nih.gov/ pubmed/18668265>. Acesso em: 06 dez. 2012. doi: 10.1007/ s00442-008-1113-z.

BAER, B. et al. Sperm storage induces an immunity cost ants. Nature, v. 441 p.872-875, 2006. Disponível em: <http://www. nature.com/nature/journal/v441/n7095/abs/nature04698.html>. Acesso em: 06 dez. 2012. doi: 10.1038/nature04698.

CAMARGO, R.S. et al. Digging effort in leaf-cutting ant queens (Atta sexdens rubropilosa) and its effects on survival and colony growth during the claustral phase. Insectes Sociaux, v.58, p.1722, 2011. Disponível em: <http://www.bv.fapesp.br/pt/producaocientifica/29563/digging-effort-leaf-cutting-ant/http://dx>. Acesso em: 06 dez. 2012. doi: 10.1007/s00040-010-0110-5.

CHAPMAN R.F. The insects: structure and function. Cambridge: Cambridge University, 1998. 770p.

COOK, S.C.et al. Colony-level macronutrient regulation in ants: mechanisms, hoarding and associated costs. Animal Behaviour, v.79, p.429-437, 2010. Disponível em: <http://journals2. scholarsportal.info/details.xqy?uri=/00033472/v79i0002/429_ cmriamhaac.xml>. Acesso em: 06 dez. 2012. doi:10.1016/j. anbehav.2009.11.022.

CUNHA, W.H.A. Observações acêrca do comportamento da iça Atta sexdens rubropilosa Forel, 1908 (Hymenoptera: Formicidae) na fundação do formigueiro. Ciência e Cultura, v.20, p.233-234, 1968.

DELLA LUCIA, T.M.C.et al. Perda de peso de rainhas de Atta durante a fundação e o estabelecimento das colônias. Revista Brasileira Biologia, v.55, n.4, p.533-536, 1995.

DELLA LUCIA T.M.C. et al. Egg-laying in Atta sexdens rubropilosa, under laboratory conditions. In: VANDER MEER R.K.; JAFFE, K. (Eds.). Applied Myrmecology - A world perspective. Basileia 1990. p.173-179.

EIDMANN, H. Zur Kenntnis der Blattschneiderameise Atta sexdens L., insbesondere ihrer Ökologie. Zeitschrift Angewandte Entomologie, v.22, p.185-436, 1935.

FRÖHLE, K.; ROCES F. The determination of nest depth in founding queens of leaf-cutting ants (Atta vollenweideri): idiothetic and temporal control. Journal of Experimental Biology, v.10 p.1642-1650. 2012. Disponível em: <http://www.ncbi.nlm.nih. gov/pubmed/22539731>. Acesso em: 06 dez. 2012. doi: 10.1242/ jeb.066217.

FUJIHARA, R.T. et al. Lipids and energy contends in the bodies of queens of Atta sexdens rubropilosa Forel (Hymenoptera: Formicidae): pre and post nuptial flight. Revista Brasileira de Entomologia, v.56,n.1,p.73-75,2012. Disponívelem: <http://www.scielo. br/scielo.php?script=sci_arttext\&pid=S0085-56262012000100011>. Acesso em: 06 dez. 2012. doi: http://dx.doi.org/10.1590/S008556262012005000015 .

JUTSUM, A.R.; QUINLAN, R.J. Flight and substrate utilisation in laboratory-reared males of Atta sexdens. Journal Insect Physiology, v.24, p.821-825, 1978. Disponível em: <http:// www.sciencedirect.com/science/article/pii/0022191078901026>. Acesso em: 06 dez. 2012. doi: http://dx.doi.org/10.1016/00221910(78)90102-6.

PASSERA, L. et al. Carbohydrates as energy source during the flight of sexuals of the ant Formica lugubris (Hymenoptera: Formicidae). Entomologial Generalis, v.15, p.25-32, 1989.

PASSERA, L.; KELLER, L. Loss of mating flight and shift in the pattern of carbohydrate storage in sexuals of ants (Hymenoptera; Formicidae). Journal of Comparative Physiology, v.160, p.207-211, 1990. Disponível em: <http://link.springer.com/ article/10.1007\%2FBF00300955>. Acesso em: 06 dez. 2012. doi: 10.1007/BF00300955.

RIBEIRO, F.J.L. Um estudo sobre o comportamento da fêmea durante a fundação da colônia em Atta sexdens rubropilosa Forel, 1908 (Hymenoptera-Formicidae). 1972. 135f. Tese (Doutorado em Psicologia) - Universidade São Paulo, SP.

SEAL, J.N. Scaling of body weight and fat content in fungus gardening ant queens: does this explain why leaf-cutting ants found claustrally? Insectes Sociaux, v.56, p.135-141, 2009. Disponível em: <http:// link.springer.com/article/10.1007\%2Fs00040-009-0002-8?LI=true>. Acesso em: 06 dez. 2012. doi: 10.1007/s00040-009-0002-8. 